\title{
How "social" is the social Simon effect?
}

\section{Thomas Dolk ${ }^{1 *}$, Bernhard Hommel ${ }^{2}$, Lorenza S. Colzato ${ }^{2}$, Simone Schütz-Bosbach ${ }^{3}$, Wolfgang Prinz $^{1}$ and Roman Liepelt ${ }^{1,4}$}

1 Department of Psychology, Max Planck Institute for Human Cognitive and Brain Sciences, Leipzig, Germany

2 Cognitive Psychology Unit, Leiden Institute for Brain and Cognition, Leiden University, Leiden, Netherlands

3 Independent Research Group "Body and Self," Max Planck Institute for Human Cognitive and Brain Sciences, Leipzig, Germany

4 Department of Psychology, Junior Group "Neurocognition of Joint Action", Westfälische Wilhelms-University, Münster, Germany

\section{Edited by:}

Diane Pecher, Erasmus University

Rotterdam, Netherlands

Reviewed by:

Klaus Kessler, University of Glasgow,

UK

Andrew D. Wilson, University of Leeds, UK

*Correspondence:

Thomas Dolk, Department of

Psychology, Max Planck Institute for

Human Cognitive and Brain Sciences,

Stephanstraße 1A, 04103 Leipzig,

Germany.

e-mail:dolk@cbs.mpg.de
In the standard Simon task, participants carry out spatially defined responses to non-spatial stimulus attributes. Responses are typically faster when stimulus location and response location correspond. This effect disappears when a participant responds to only one of the two stimuli and reappears when another person carries out the other response. This social Simon effect (SSE) has been considered as providing an index for action co-representation. Here, we investigated whether joint-action effects in a social Simon task involve mechanisms of action co-representation, as measured by the amount of incorporation of another person's action. We combined an auditory social Simon task with a manipulation of the sense of ownership of another person's hand (rubber hand illusion). If the SSE is established by action co-representation, then the incorporation of the other person's hand into one's own body representation should increase the SSE (synchronous > asynchronous stroking). However, we found the SSE to be smaller in the synchronous as compared to the asynchronous stroking condition (Experiment 1), suggesting that the SSE reflects the separation of spatial action events rather than the integration of the other person's action. This effect is independent of the active involvement (Experiment 2) and the presence of another person (Experiment 3). These findings suggest that the "social" Simon effect is not really social in nature but is established when an interaction partner produces events that serve as a spatial reference for one's own actions.

Keywords: joint action, social Simon, social cognition, rubber hand illusion

\section{INTRODUCTION}

Many activities we perform in daily life are carried out together with other people. But how do we mentally represent other people's actions and how does this affect our own behavior?

Recent research suggests that joint action can lead to the representation of one's own and other's actions. This "action co-representation" is thought to facilitate action prediction and coordination of one's own actions with those of others (Sebanz et al., 2006). Evidence for this view stems from the "social Simon task" developed by Sebanz et al. (2003). In the standard Simon task (Simon and Rudell, 1967; Simon, 1990), participants typically carry out spatially defined responses (e.g., left and right key presses) to non-spatial stimulus attributes (e.g., auditory pitch or visual color) that randomly appear on the left or right. For example, participants are required to press a right key whenever they perceive a high-pitched tone and a left key in response to a low-pitched tone. Although stimulus location is completely irrelevant in this task, responses are typically faster when they spatially correspond to the stimulus signaling them. That is, spatial stimulus-response compatibility facilitates task performance, a phenomenon that has come to be known as the Simon effect. Commonly, this effect disappears when a participant responds to only one of the two stimuli, rendering the task a "go-nogo task" (Hommel, 1996). However, if the same go-nogo task is shared between two participants so that each of them operates one of the two responses, a Simon effect is observed (Sebanz et al., 2003) - the "social Simon effect" (SSE).
According to the dimensional overlap model (Kornblum et al., 1990) the standard Simon effect can be explained by a match between the spatially irrelevant dimension of the stimulus and the relevant response dimension (Hommel et al., 2001). Accordingly, responses are assumed to be automatically activated if the stimulus spatially corresponds to the correct response and thus facilitate task performance, whereas a lack of correspondence between stimulusresponse pairs leads to response competition.

It is fair to say that the mechanisms underlying the SSE are poorly understood. Some authors have claimed that, due to the fundamentally social nature of perception and action, people automatically co-represent other people's actions (Knoblich and Sebanz, 2006). However, a finding that does not seem to be completely in line with the idea of action co-representation being social, automatic, and mandatory is that the SSE is fully present in autistic participants (Sebanz et al., 2005), who can be assumed to have difficulties processing social information. According to Guagnano et al. (2010), the major role of the co-actor in the social Simon task might be to provide a spatial reference frame that allows coding of one's own action as left or right relative to the other person just as one's own action alternatives provide a reference frame for relative response coding (Hommel, 1996). Guagnano et al. (2010) further claimed that this reference frame can only be used if the other person is located within a participant's peripersonal space. In line with a spatial reference explanation for the SSE, the authors were able to show that the SSE breaks down if the two co-actors 
are seated outside of arm's reach. However, this approach does not easily explain why an individual's bad mood (Kuhbandner et al., 2010) or negative relationship with the co-actor (Hommel et al., 2009) eliminates the effect.

In the present study, we make a further attempt to clarify what the notion of action co-representation might mean, what it refers to, and in which sense it might account for the SSE. In essence, it may be possible to distinguish between three concepts of action co-representation, ranging from strong to weak. According to the first, strong concept, the SSE is assumed to be functionally similar to the effect obtained when one person is taking care of both responses (Sebanz et al., 2003). Following this line of reasoning, the SSE is due to the cognitive integration of the co-actor and his/ her actions into the actor's body scheme. The second, intermediate concept, assumes that actors represent information about their co-actor and his/her actions without integrating it with representations of their own body and actions. This co-representation of the self and other provides a reference frame for the (e.g., spatial) coding of an individual's own actions relative to the other person and his/her actions (Guagnano et al., 2010). Thus, rather than incorporating the other person into the actor's body schema, the co-actor is represented as a social agent responsible for the alternative action separately from one's own body and action. According to the third, weak concept, the co-actor does not function as a social being but mainly by virtue of producing particular events (actions with perceivable effects), which serve as reference for coding one's own action.

Our experiments proceeded from testing the strongest to the weakest concept. In Experiment 1, we tested whether the SSE is affected by the perceived ownership of another person's hand as suggested by a strong conceptualization of action co-representation (Sebanz et al., 2003; Knoblich and Sebanz, 2006). A reliable paradigm to experimentally manipulate the sense of ownership of another person's hand is the rubber hand illusion (RHI; Botvinick and Cohen, 1998). Here, a rubber hand (or another person's hand) is stroked either synchronously or asynchronously. During synchronous stroking, the subject commonly feels the illusion that the seen rubber (or foreign) hand becomes a part of his/her own body.

We experimentally combined the RHI with an auditory social Simon task. In Experiments 2 and 3, we gradually de-socialized the task situation. In Experiment 2, we tested if we could find evidence of a SSE without the active involvement of the co-actor. In Experiment 3, we excluded the co-actor from the task setting altogether to test the weak concept of action co-representation.

\section{EXPERIMENT 1}

The aim of Experiment 1 was to investigate whether the SSE relies on or varies as a function of action co-representation induced by the RHI. Participants performed an auditory social Simon task while the perceived ownership of another person's hand (i.e., synchronous vs. asynchronous stroking) was manipulated.

The RHI is assumed to arise from a multimodal conflict between vision, touch, and proprioception (Ehrsson et al., 2004; Tsakiris and Haggard, 2005; Kammers et al., 2009). As vision usually dominates touch and proprioception (Constantini and Haggard, 2007), the RHI emerges as a consequence of synchronous but not asynchronous stroking. When stroking is synchronous, the sense of owner- ship is strong. As a result, the activity of the other hand should be more strongly attributed to one's own body and thus induce an integration of another person into one's own action representation. Conversely, in the asynchronous stroking condition, the other hand is more likely to be attributed to a different actor (Botvinick and Cohen, 1998) and thus, clearly separated from one's own action. This condition was hypothesized to work against the strong concept of action co-representation.

If the SSE relies on the cognitive integration of the co-actor and his/her actions into the actor's body schema (strong concept), synchronous stroking should create a more pronounced SSE compared to asynchronous stroking. However, if an actor tends to represent the co-actor as separate from him/herself and not integrate the other's actions into their own body schema (intermediate concept), synchronous stroking might actually lead to a smaller, rather than a larger SSE than asynchronous stroking does. This is because the asynchronous stroking might increase the saliency of the other person's hand and its actions, and thereby provide a stronger spatial reference for coding the actor's own action.

\section{METHODS}

\section{Participants}

Forty healthy undergraduate students (20 female; 20-25 years of age, mean age $=23.8$ ) with no history of neurological or hearing problems participated in Experiment 1. Twenty served as actual participants (henceforth called actors) and 20 as co-actors (see Figure 1). The participants were all right-handed as assessed by the Edinburgh Inventory (Olfield, 1971), had normal or correctedto-normal vision, were naive with regard to the hypothesis of the experiment and were paid $€ 14$ for participating.

\section{Apparatus and stimuli}

An auditory Simon task (go-nogo task) was used. In each trial, one of two sounds designed by van Steenbergen (2007) and chosen as go (sound A) and nogo (sound B) was presented via two loudspeakers separated by a distance of $1 \mathrm{~m}$ at approximately $60 \mathrm{~dB}$ to either the left or right side of both participants.

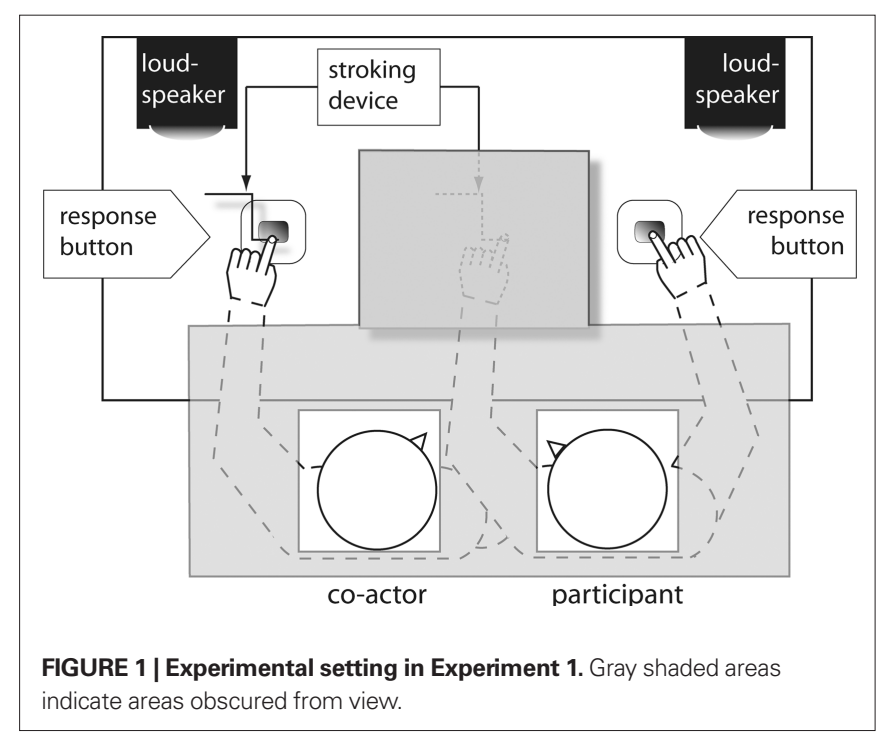


To experimentally induce a sense of ownership of the other person's hand, we made use of the RHI. This involved stimulating the actor's and the co-actor's hand mechanically by means of two computer-controlled stepper motors, each with two identical paintbrushes attached, allowing the precise control of onset, direction, speed, and duration of both steppers independently. Following Lloyd (2007), the distance between both stroking devices was about $22.5 \mathrm{~cm}$.

\section{Subjective measures}

Participants rated the perceived strength of the RHI by working through nine statements directly after each induction and experimental phase. The statements were translated from the original RHI Questionnaire (Botvinick and Cohen, 1998) and participants were to agree or disagree on a visual analog scale from left $(0=$ "completely disagree") to right $(10=$ "completely agree"). The first three statements are suggested to capture the core of the illusion (Botvinick and Cohen, 1998; Schütz-Bosbach et al., 2008; Kammers et al., 2009): (1) "It seemed as if I were feeling the touch of the paintbrush in the location where I saw the rubber hand touched"; (2) "It seemed as though the touch I felt was caused by the paintbrush touching the rubber hand"; (3) "I felt as if the rubber hand were my hand." A successful RHI induction would be indicated by higher ratings after synchronous than asynchronous visual-tactile stimulation.

\section{Task and procedure}

The experiment consisted of two consecutive sessions, each including an induction and an experimental phase. To avoid carryover effects, both sessions were separated by a 5-min mandatory break. Prior to the induction phase, participants were seated next to each other. The actual participant (see Figure 1) was always seated on the right and was asked to place his/her left index finger under the stroking device, so that the paintbrush could stimulate the occluded index finger from the knuckle to the fingertip or vice versa. His/her right index finger rested on the right response button. Randomly chosen co-actors, whose performance was not analyzed, were always seated on the left. They rested their left index finger on the left response button ( $80 \mathrm{~cm}$ between the two response buttons) directly under the left stroking device and their right hand on their lap under the table. After participant and co-actor were seated and had placed their hands in the correct positions, a white towel was placed over their shoulders and arms to obscure everything on the table except the co-actor's left and the participant's right hand (see Figure 1).

The experiment started with the induction phase. The stimulation was delivered mechanically by two stepper motors to which paintbrushes were attached. The amount of stimulation (onset, direction, speed, and duration) was precisely matched across conditions. To avoid habituation effects, the speed and direction of the paintbrushes were unpredictable and changed randomly every $5 \mathrm{~s}$. In the synchronous condition, the participant's and the coactor's left index fingers were stroked in synchrony, with identical location, timing, and trajectory parameters. In the asynchronous condition, the parameters differed between the two stroked fingers, while the total amount of stimulation for both index fingers was the same as in the synchronous condition. Thus, the synchronous

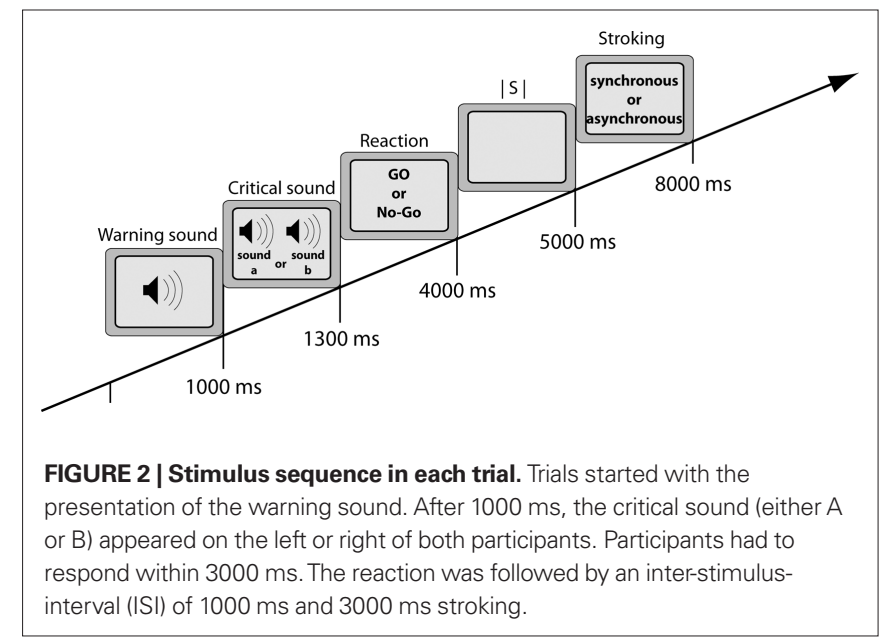

and asynchronous stroking conditions differed only in the phase of the temporal structures of visual and tactile stimulation. The stroking procedure in each induction phase lasted for about 3-min. After the stimulation, both participant and co-actor were asked to fill out the RHI Questionnaire.

After completing the questionnaires, the experimental phase started. There were four blocks of 64 trials for each participant and co-actor (32 with spatially compatible stimulus-response relationships and 32 with spatially incompatible relationships). Each trial began with the presentation of the warning sound. After $1000 \mathrm{~ms}$, the critical sound - either sound A or B - was presented to the right or the left side of both the participant and co-actor, requiring a response as quickly and as accurately as possible. Participant and co-actor were instructed to fixate on the other's hand and to respond exclusively to the sound assigned to them, irrespective of its location. Each response was followed by a $1000 \mathrm{~ms}$ interstimulus-interval (ISI) and $3000 \mathrm{~ms}$ stroking, which was always congruent to the stroking type of the corresponding induction period (either synchronous or asynchronous) to refresh the RHI (see Figure 2).

Feedback [mean reaction time (RT) and percentage correct] as well as a 2-min break were provided at the end of each block. After completing the first four blocks, participants were asked to fill in the RHI Questionnaire again, which was followed by a 5-min break to avoid carryover effects to the second session of the experiment. After the break, the second session started. The procedure was the same as in the first session except for the type of stimulation, which was always different from that in the first session. The order of stimulation type (synchronous followed by asynchronous stroking or vice versa) was counterbalanced across participants.

\section{RESULTS}

In the following, only data from the actual participants (actors) were analyzed.

\section{Rubber hand questionnaire}

Participants experienced the co-actor's hand as their own hand as a consequence of synchronous but not asynchronous stroking during both the induction and experimental phase: The RHI was significantly stronger after synchronous than after asynchronous 
stroking (RHI-related questions 1-2 after the induction and 1-3 after the experimental phase; two-way paired-sample $t$-tests; all ps $<0.05)$.

\section{Simon task}

Reaction times. Responses were coded as compatible (stimulus ipsilateral to the correct response side) and incompatible (stimulus contralateral to the correct response side). Mean RTs on the auditory social go-nogo Simon task for the 20 actual participants were submitted to a 2 (Compatibility: compatible, incompatible) $\times 2$ (Stroking: synchronous, asynchronous) within-subjects repeated measures analysis of variance (ANOVA). The analysis showed a significant main effect of Compatibility $\left[F(1,19)=25.46, p<0.001, \eta^{2}=0.57\right]$ indicating that responses were faster with spatially compatible (mean $\mathrm{RT}=291 \mathrm{~ms}$ ) than with incompatible stimulus-response relationships (mean RT = $313 \mathrm{~ms}$ ). More importantly, the compatibility effect varied with stroking, as indicated by a significant interaction of Compatibility $\times$ Stroking $\left[F(1,19)=5.88, p<0.05, \eta^{2}=0.24\right.$; see Figure 3]. The $29 \mathrm{~ms}$ compatibility effect observed in the asynchronous stroking condition was significantly larger $[F(1,19)=25.17, p<0.001$, $\left.\eta^{2}=0.57\right]$ than the $15 \mathrm{~ms}$ compatibility effect in the synchronous stroking condition $\left[F(1,19)=10.82, p<0.01, \eta^{2}=0.36\right.$; see Figure 3]. The main effect of Stroking was not significant $\left[F(1,19)<1, \eta^{2}=0.01\right]$. To check for possible task order effects, we performed an additional ANOVA with Order as a between-subjects factor - but the three-way interaction was not significant $\left[F(1,18)=1.40, p>0.05, \eta^{2}=0.07\right]$.

Error rates. We observed a significant main effect of Compatibility $\left[F(1,19)=12.67, p<0.01, \eta^{2}=0.40\right]$, indicating higher error rates for incompatible $(1.0 \%)$ than for compatible trials $(0.3 \%)$. The interaction of Compatibility $\times$ Stroking was far from significance $\left[F(1,19)<1, \eta^{2}=0.01\right]$, which rules out a speed-accuracy trade-off.

\section{DISCUSSION}

The aim of this experiment was to test predictions of a strong concept of action co-representation accounting for the SSE. In particular, we investigated whether the SSE is mediated by the degree to which the active hand of a co-actor is perceived to be a part of the actor's own body.

First of all, we were able to replicate the findings of Sebanz et al. (2003), confirming that our particular setup was sufficiently sensitive to elicit the SSE in both the synchronous and the asynchronous

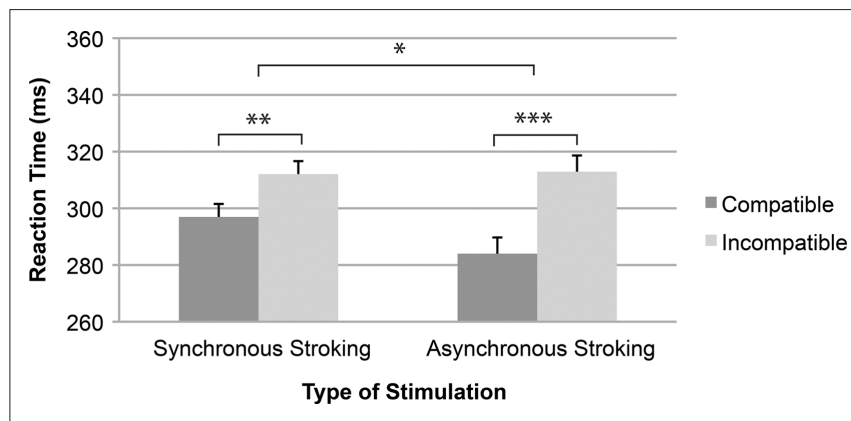

FIGURE 3 | Mean reaction time as a function of the type of stimulation and spatial stimulus-response compatibility. Error bars represent standard errors of the mean differences. ${ }^{*} p<0.05,{ }^{* *} p<0.01,{ }^{* *} p<0.001$. stroking conditions. Second, we found the effect to be smaller, rather than larger, with synchronous than with asynchronous stroking. Thus, the incorporation of another person's hand into one's own body schema through the RHI (induced by synchronous stroking) reduces the SSE as compared to a condition where the co-actor is represented as a separate actor (induced by asynchronous stroking). This interpretation is supported by the subjective rating of the sense of ownership of the co-actor's hand in the synchronous stroking condition, which indicates that the experimental RHI manipulation was successful across all phases of the experiment.

These results provide considerable evidence against a strong concept of action co-representation as a mechanism underlying the SSE. That is, the SSE seems to occur even though actors represent their own action and the action of their co-actor separately. Emphasizing the difference between the two actions - or the related effectors - leads to a more pronounced SSE. This increase of the SSE in the asynchronous stroking condition is in line with the assumption that the SSE is established by the coding of one's action in reference to other actions (intermediate concept) or salient events (weak concept). Referential coding is known to be a basic principle operating in the Simon task (Hommel, 1993). Stimuli have been shown to be spatially coded relative to other stimuli that are either voluntarily attended to (Nicoletti and Umiltà, 1989) or that are salient enough to attract attention involuntarily (Treccani et al., 2006). With respect to action, response location has been shown to be coded in reference to other possible or recent responses (Hommel, 1996), in particular on spatial dimensions that help to discriminate between response alternatives (Ansorge and Wühr, 2004).

Given that most authors agree that the Simon effect is due to some sort of match or mismatch between spatial stimulus and response codes (Kornblum et al., 1990; Prinz, 1990; Hommel et al., 2001), the effect can only occur if stimulus location and response location are coded on the same dimension - as left and right in our case. In a standard Simon task, where the same participant performs both responses, this is very likely to happen, as the left-right dimension is particularly salient and provides the best discriminability between the two responses. In the social Simon task, however, participants operate only one response, so there is no actual need for spatial coding. Yet, if a co-actor (or perhaps another event) is sufficiently salient, people may nevertheless tend to code their response in reference to the spatial location of the other person or event (cf. Guagnano et al., 2010).

According to this reasoning, the social aspect of the joint-action situation created by the social Simon task may be just one of perhaps many factors that attract attention to other events and thereby induce the referential coding of one's own action, thus creating or enhancing the SSE. One implication of this possibility is that the active involvement of the co-actor in the present task might not necessarily induce referential response coding and elicit the SSE. To test this possibility, we performed a second experiment that included a now inactive but still salient "co-actor."

\section{EXPERIMENT 2}

The aim of Experiment 2 was to test whether the SSE can also be obtained with an inactive co-actor (to whom we will nevertheless keep referring to as "co-actor" for the sake of convenience). To do 
so, we replicated Experiment 1 but now de-socialized the task to some degree: The co-actor no longer responded but sat passively next to the actual participant. If the co-actor provides a spatial reference frame for the coding of one's own action as left or right relative to the other person, one should expect a SSE even with an inactive co-actor. By contrast, however, if the active participation of the co-actor as a responding agent is crucial for the SSE to emerge as the original approach of Guagnano et al. (2010) suggests, the Simon effect should disappear.

\section{METHODS \\ Participants}

Twenty new healthy undergraduate students ( 10 female; $21-30$ years of age, mean age $=24.8$ ) with no history of neurological or hearing problems participated in Experiment 2. They fulfilled the same criteria and were treated in the same way as the participants in Experiment 1 .

\section{Apparatus, stimuli, task, and procedure}

These were the same as in Experiment 1, with the following exceptions. The co-actor, who was the same for all participants, sat alongside the actual participant, and was no longer actively involved in the task. The left response button and the stroking device were visible on the co-actor's left side (see Figure 4). The co-actor was instructed to watch the behavior of the participant.

\section{RESULTS}

\section{Reaction times}

The 2 (Compatibility: compatible, incompatible) $\times 2$ (Stroking: synchronous, asynchronous) within-subjects repeated measures ANOVA revealed a significant main effect for Compatibility $[F(1,19)=14.05$, $\left.p<0.005, \eta^{2}=0.43\right]$, showing that responses were faster with stimulus-response compatibility (mean RT $=335 \mathrm{~ms}$ ) than with stimulusresponse incompatibility (mean $\mathrm{RT}=347 \mathrm{~ms}$ ). The effects of Stroking $\left[F(1,19)<1, \eta^{2}=0.03\right]$ and the Compatibility $\times$ Stroking interaction $\left[F(1,19)<1, \eta^{2}=0.01\right.$; see Figure 5] were not significant. An additional ANOVA with Order as a between-subjects factor revealed no significant interaction $\left[F(1,18)=1.66, p>0.05, \eta^{2}=0.08\right]$.

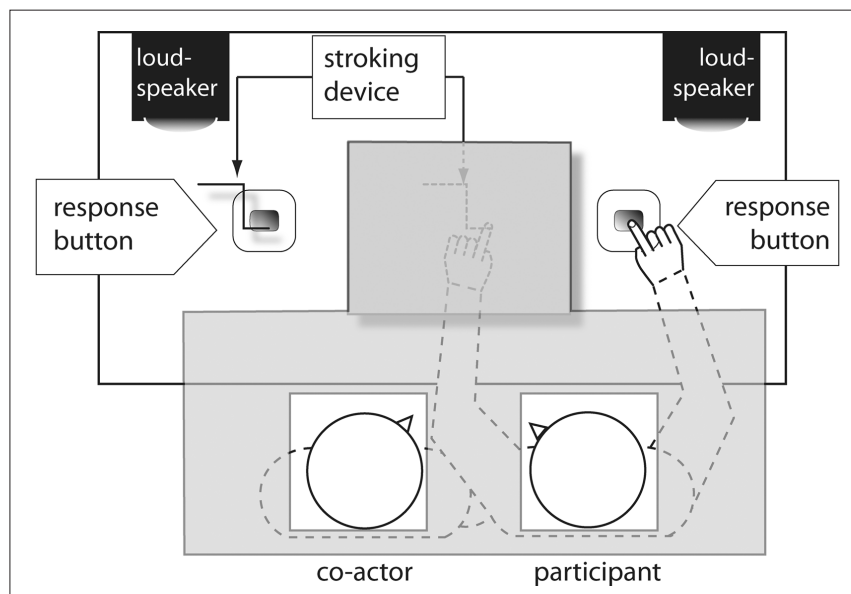

FIGURE 4 | Experimental setting in Experiment 2. Gray shaded areas indicate areas obscured from view.

\section{Error rates}

Neither the effects of Compatibility $[F(1,19)=2.49, p>0.05$, $\left.\eta^{2}=0.12\right]$ and Stroking $\left[F(1,19)<1, \eta^{2}=0.01\right]$, nor the interaction of Compatibility $\times$ Stroking were significant $[F(1,19)=1.15$, $\left.p>0.05, \eta^{2}=0.06\right]$.

\section{DISCUSSION}

Experiment 2 was designed to investigate whether the SSE can be obtained independent of the active involvement of another person. We found a significant overall Simon effect, which did not vary with the type of stroking. Extending previous findings (Guagnano et al., 2010), the present results suggest that the SSE can be established irrespective of what the other person is doing and whether this person is actively involved in the same or any other task.

This provides evidence against the interpretation of the SSE as a genuine joint-action effect (Sebanz et al., 2006) or as evidence for shared task representations (Knoblich and Sebanz, 2006; Ruys and Aarts, 2010). It also challenges the claim that it is "the presence of an active confederate" that provides the crucial reference for coding one's own action in space (Guagnano et al., 2010). This is not to say that the activity of the co-actor in Experiment 1 played no role at all. For one, the size of the SSE under asynchronous stroking was significantly smaller in Experiment $2(13 \mathrm{~ms})$ than it was in Experiment 1 [ $29 \mathrm{~ms} ; F(1,38)=5.48, p<0.05, \eta^{2}=0.13$ ] and, for another, the type of stroking affected the size of the SSE in Experiment 1 but not in Experiment 2. This suggests that the representation of one's own response is equipped with a spatial feature (right) if a second person is actively engaged in the task (Experiment 1) or if a non-social event, such as the movement of the stroking device, is present (Experiment 2). Thus, once another action alternative is sufficiently salient (e.g., the movement of the stroking device in the other response dimension), one's own action seems to be coded with reference to it, thus favoring the weak concept of action co-representation.

However, the finding of a Simon effect in Experiment 2 might alternatively be explained by the assumption that the mere presence of another person provided a reference for the coding of the alternative action event to one's own action (intermediate concept). In order to test this alternative explanation, we performed a third experiment.

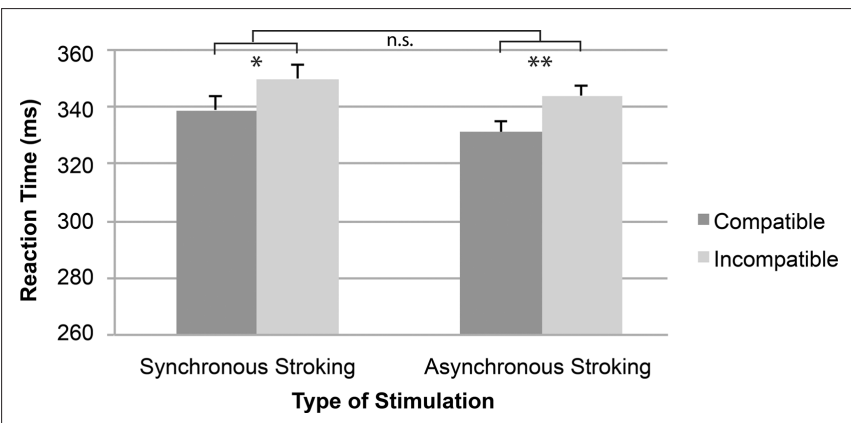

FIGURE 5 | Mean reaction time as a function of the type of stimulation and spatial stimulus-response compatibility. Error bars represent standard errors of the mean differences. ${ }^{*} p<0.05,{ }^{* *} p<0.01$, n.s., not significant. 


\section{EXPERIMENT 3}

The aim of Experiment 3 was to investigate the role of the presence of another person in the SSE. According to the weak concept of action co-representation, the co-actor does not function as a social actor but mainly as a source that produces particular events, which then serve as reference for coding one's own action. Guagnano et al. (2010) suggests that spatial response coding would be prompted by the presence of another active person in peripersonal space, irrespective of whether this person is working on the same task or not. Even though the present Experiment 2 suggests that this other person can just as well be inactive, the presence of a person may still be relevant. However, another possibility is that, even though the presence of another person is a particularly salient event, any salient event - social as well as non-social - could propagate referential coding (weak concept). With respect to our experimental setup, the mere manipulation of stroking might be sufficient to establish a salient non-social event that induces referential coding.

To test the latter hypothesis, we repeated Experiment 2 by further de-socializing the task: We no longer included another person. In one condition, the experimental procedure was the same as in Experiment 2, including the stroking device and the stroking manipulation (the "Device present" condition). This manipulation was expected to establish a salient event on the left side that could serve as a landmark for the participant to code his or her action as "right." If so, a SSE would be expected. In the other condition (the "Device absent" condition, see Figure 6), the stroking device on the participant's left was no longer present. However, the device above the participant's occluded left hand was still there, but there was no stroking manipulation any more, thus providing no landmark for referential coding.

\section{METHODS}

\section{Participants}

Twenty new healthy undergraduate students ( 11 female; $20-30$ years of age, mean age $=24.9)$ with no history of neurological or hearing problems participated in Experiment 3. They fulfilled the same criteria and were treated in the same as the participants in Experiments 1 and 2 .

\section{Apparatus, stimuli, task, and procedure}

These were the same as in the previous experiments, with the following exceptions. Participants carried out the task alone, in the absence of any other person, either with the moving stroking device ("Device present") or without the stroking device ("Device absent") operating at the alternative response side (see Figure 6).

The two conditions "Device present" and "Device absent" were manipulated within participants. In the "Device present" condition, the movements of the device stroking the participant's left hand and the visible device on the left side of the table were asynchronous. During the "Device absent" condition, both the response button and the stroking device on the participant's left were removed. However, to keep all other factors consistent with the previous experiments, the device above the participant's occluded left hand was still present, but no longer stimulated the hand (see Figure 6). The participants either started with the "Device present" or the "Device absent" condition; the order was counterbalanced across participants. Participants were seated on the right chair throughout the whole experiment and had to respond with their right index finger, pressing the right button. They were instructed to respond only to the tone assigned to them irrespective of the location and had to fixate on either the stroking device ("Device present") or a similar point on the empty table ("Device absent"; see Figure 6).

\section{RESULTS}

\section{Reaction times}

A 2 (Compatibility: compatible, incompatible) $\times 2$ (Device: device present, device absent) within-subjects repeated measures ANOVA revealed a significant interaction of Compatibility $\times$ Device $\left[F(1,19)=4.54, p<0.05, \eta^{2}=0.19\right.$; see Figure 7]. Follow-up analyses confirmed that the compatibility effect observed in the "Device present" condition ( $9 \mathrm{~ms}$ ) was significant $[F(1,19)=5.53$, $p<0.05, \eta^{2}=0.23$ ], whereas the reversed compatibility effect in the "Device absent" condition ( $-7 \mathrm{~ms}$, Compatible: mean RT $=314 \mathrm{~ms}$; Incompatible: mean $\mathrm{RT}=307 \mathrm{~ms})$ was not $\left[F(1,19)<1, \eta^{2}=0.05\right.$; see Figure 7]. Participants responded faster with stimulusresponse compatibility (mean RT $=304 \mathrm{~ms}$ ) than with stimulus-response incompatibility (mean RT $=313 \mathrm{~ms}$ ) in the "Device present" condition, whereas the RTs were slower for compatible
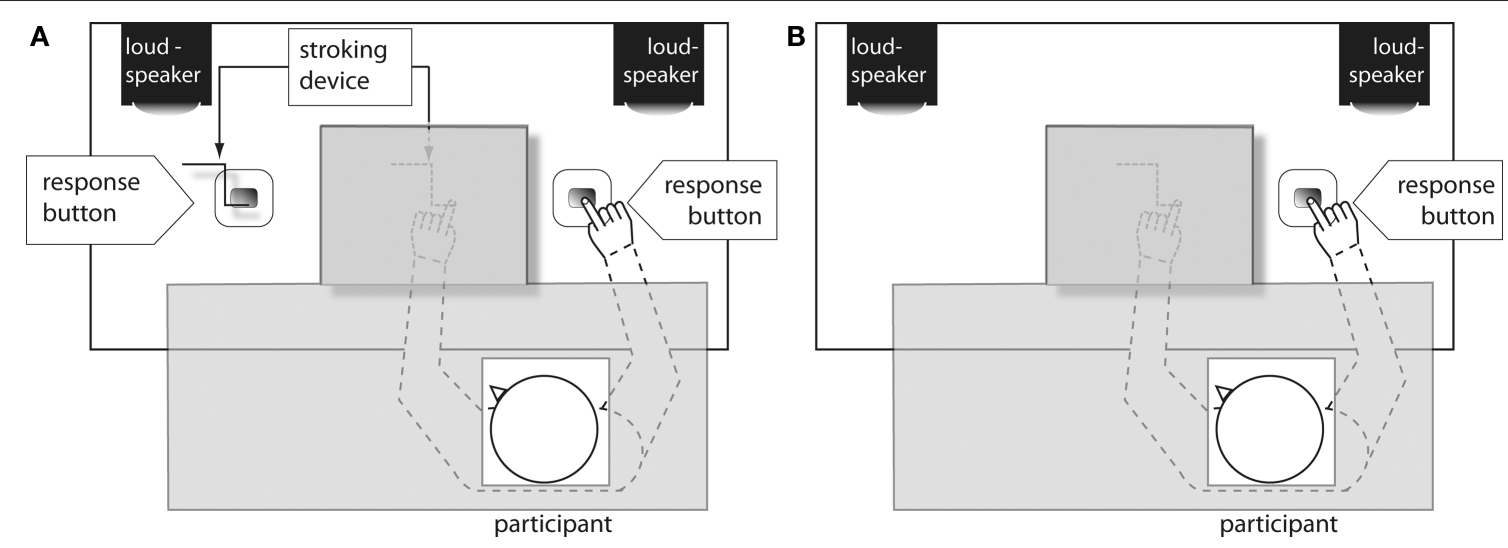

FIGURE 6 | Experimental setting in Experiment 3, in the "Device present" (A) and the "Device absent" condition (B). Gray shaded areas indicate areas obscured from view. 


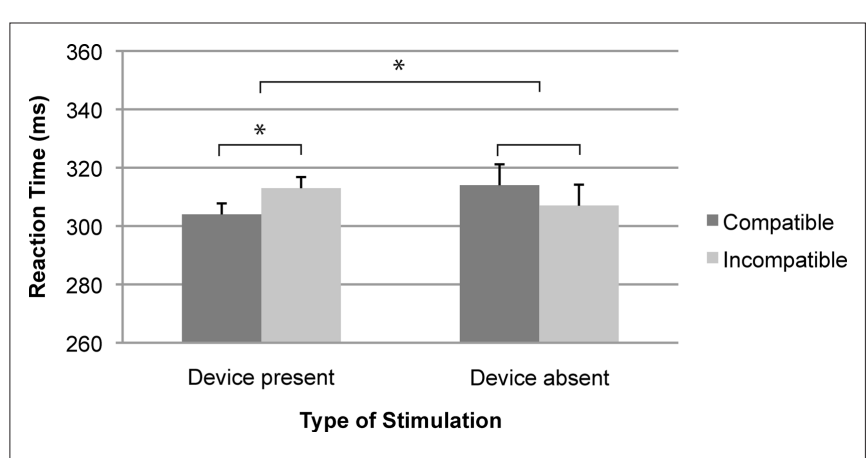

FIGURE 7 | Mean reaction time as a function of the type of stimulation and spatial stimulus-response compatibility. Error bars represent standard errors of the mean differences. ${ }^{*} p<0.05$.

trials (mean RT $=314 \mathrm{~ms}$ ) compared to incompatible trials (mean $\mathrm{RT}=307 \mathrm{~ms}$ ) in the "Device absent" condition. The effects of Compatibility and Device were not significant (all Fs $<1$ ). An additional ANOVA with Order as a between-subjects factor revealed no interaction between Compatibility $\times$ Device $\times$ Order $[F(1,18)<1$, $\left.\eta^{2}=0.02\right]$.

\section{Error rates}

Neither the effects of Compatibility $\left[F(1,19)=1.72, p>0.05, \eta^{2}=0.08\right]$ and Device $\left[F(1,19)=2.26, p>0.05, \eta^{2}=0.11\right]$, nor the interaction of Compatibility $\times$ Device were significant $\left[F(1,19)<1, \eta^{2}=0.01\right]$.

\section{DISCUSSION}

To disentangle whether it was the other person or the movement of the stroking device that acted as a reference frame for referential response coding, we repeated Experiment 2 without another person present. We found a significant Simon effect in the "Device present" condition but not in the "Device absent" condition, which suggests that the stroking device and/or its activities provided a reference for the spatial coding of the participant's own actions.

Given the rather small effects we obtained, these findings need to be handled with caution. Nevertheless, the interaction was clearly reliable and the sign of the SSE reversed in the "Device absent" condition. Moreover, the relatively modest size of the SSE in Experiment 3 fits well with the observations from other studies (e.g., Guagnano et al., 2010, Experiment $1=7$ ms, Experiment $2 \mathrm{~b}=5 \mathrm{~ms}$; Liepelt et al., $2011=9 \mathrm{~ms}$; Sebanz et al., $2003=8 \mathrm{~ms}$ ), suggesting that the present results are within a comparable range. Accordingly, we take these findings as evidence that the SSE can be obtained under entirely non-social circumstances.

To test whether the presence of the other person added to the effect in Experiment 2, we combined the data from Experiments 2 and 3 and performed an ANOVA with Compatibility (compatible, incompatible) as a within-subjects factor and Experiment (Experiment 2 - asynchronous stroking condition, Experiment 3- "Device present" condition) as a between-subjects factor. This analysis revealed no significant interaction, suggesting that another person is neither necessary nor particularly relevant to provide a spatial reference for coding one's own action. Even a non-social salient event seems to have the potential to affect the way people represent their action and, thereby, to produce a (no longer so social) Simon effect.

\section{GENERAL DISCUSSION}

The present study was designed to clarify what the notion of action corepresentation might be taken to mean and whether it is necessary for an account of the SSE. By experimentally inducing the RHI in an auditory social Simon task (in Experiment 1), we investigated whether the SSE is mediated by the degree to which actors incorporate other people's actions (or acting effectors) into their own body schema. Although we were able to replicate previous findings of Sebanz et al. (2003) and reproduced reliable RHIs in the actors, $\mathrm{RHI}$-induced co-representation of a co-actor reduced, rather than increased, the SSE. This pattern of results provides clear evidence against a strong concept of action corepresentation. Rather, asynchronous stroking apparently emphasized the existence of the co-actor, which was likely to provide a landmark for the spatial coding of the actor's response (Guagnano et al., 2010). This reasoning might be explained by the intermediate concept of action co-representation, which allows the separate representation of actor and co-actor (Hommel, 1993, 1996; Liepelt et al., 2011).

This interpretation received support from Experiment 2, where the other person was no longer actively involved in the task. In Experiment 3, however, the salient landmark was no longer a social event. In both cases, a reliable Simon effect was obtained even though - at least in Experiment 3 - the effect was clearly not a "social" effect anymore. In other words, the "SSE" can be induced through social as well as through non-social events, indicating that even an intermediate concept of action co-representation is not necessary to account for the SSE.

This assumption appears to be reasonable when considering the task requirements in the single and social Simon go-nogo task. In both cases, the spatial coding of responses is unnecessary and, as indicated by the absence of substantial Simon effects under some conditions (e.g., Hommel, 1996; Sebanz et al., 2003), apparently not obligatory either. This suggests that people are able to abstract from most aspects of their current environment and focus on what is currently relevant such as the action they are to carry out. However, irregular events are known to attract attention in a bottom-up fashion and it makes sense to assume that this is particularly true for events that are social in nature (Philipp and Prinz, 2010; Ruys and Aarts, 2010). This attraction of attention seems to be sufficient to induce the tendency or perhaps even the need to code one's action spatially in reference to this attention-attracting event.

In summary, testing predictions from three concepts of action co-representation revealed that neither the integration of another person's action into an individual's body representation (strong concept) nor the separate cognitive representation of one's own and the other person's action (intermediate concept) appear to be necessary for the SSE to occur. As even non-social events are sufficient to reliably influence an individual's own task performance (see Experiment 3), it seems to be the presence or expectation of salient events as such that underlies the SSE (Vlainic et al., 2010). Moreover, the present results suggests, that even the modality of these events does not matter much: Both proprioceptive events (resulting from the stroking of the participant's occluded hand) and visual events (resulting from the motion of the stroking device in the alternative response dimension) were functional in providing a reference frame for action coding. Identifying the factors that determine the relative saliency and the relative contributions of visual, proprioceptive, and other information to the SSE represents an important goal for future research. 
The general observation that a salient event induces referential action coding is in line with the assumptions of the theory of event coding (Hommel et al., 2001; Hommel, 2004, 2009). The presence of another person sitting next to the actor performing his/her part of the task represents a salient event that provides an alternative for the actor's own response and thus calls for perceptual discrimination between the two (e.g., in terms of "left" vs. "right" and "me" vs. "not me"; Hommel et al., 2009; Liepelt et al., 2011). Once a response is spatially coded, it provides a reference that is compatible or incompatible with respect to the assigned stimulus (Guagnano et al., 2010). Establishing or using a task-relevant feature dimension may create feature overlap (Kornblum et al., 1990; Lam and Chua, 2009) and feature-correspondence effects, which are the basis of the Simon effect and other stimulus-response compatibility effects.

Our considerations should not be taken to mean that task sharing - in the sense of considering aspects of someone else's task in one's own cognitive task representation - is not social at all, nor does it imply that social aspects play no role at all in the SSE. As already mentioned, social factors like mood (Kuhbandner et al., 2010) or interpersonal relationship (Hommel et al., 2009) have been found to affect the size and presence of the effect. Nevertheless, these effects might well be mediated by the saliency they lend to the alternative action and/or actor, and the impact of this saliency on referential response coding. Further support for the claim that the

\section{REFERENCES}

Ansorge, U., and Wühr, P. (2004). A response-discrimination account of the Simon effect. J. Exp. Psychol. Hum. Percept. Perform. 30, 365-377.

Blake, R., and Shiffrar, M. (2007). Perception of human motion. Annu. Rev. Psychol. 58, 47-73.

Botvinick, M., and Cohen, J. (1998). Rubber hands 'feel' touch that eyes see. Nature 391, 756.

Constantini, M., and Haggard, P. (2007). The rubber hand illusion: sensitivity and reference frame for body ownership. Conscious. Cogn. 16, 229-240.

Cross, E. S., Hamilton, A. F. D. C., Kraemer, D. J. M, Kelley, W. M., and Grafton, S. T. (2009). Dissociable substrates for body motion and physical experience in the human action observation network. Eur. J. Neurosci. 30, 1383-1392.

Ehrsson, H. H., Spence, C., and Passingham, R. E. (2004). That's my hand. Activity in premotor cortex reflects feeling of ownership of a limb. Science 305, 875-877.

Guagnano, D., Rusconi, E., and Umiltà, C.A. (2010). Sharing a task or sharing space? On the effect of the confederate in action coding in a detection task. Cognition 114, 348-355.

Heberlein, A. S., Adolphs, R., Tranel, D., and Damasio, H. (2004). Cortical regions for judgements of emotions and understanding of social events is grounded in visual perception is given by the extensive literature on biological motion (Cross et al., 2009; Liepelt and Brass, 2010; for a review see Blake and Shiffrar, 2007) and emotions (Pollick et al., 2001; Heberlein et al., 2004). Accordingly, the SSE is another excellent example of the understanding of social events throughout salient signals we are sensitive to. Future research should take this into account to improve our understanding of social cognition.

Taken together, the present study suggests that the social Simon may be socially induced but is not really social in nature. Rather than requiring or necessarily reflecting the co-representation of the other person's action into an individual's own body and/or task representation, the effect seems to result from salient social or non-social actions or events that induce the coding of an individual's own action as left or right - a necessary condition for the Simon effect to emerge.

\section{ACKNOWLEDGMENTS}

We thank Henrik Grunert for technical support, Rosie Wallis for editing the article from a native speaker's perspective, Patricia Grocke for being the confederate in the second experiment and Anna Schmidt for her assistance with the data collection for the third experiment. The authors wish to thank Emily Cross and the reviewers for their helpful comments on a previous draft of this article. personality traits from point-light walkers. J. Cogn. Neurosci. 16, 1143-1158.

Hommel, B. (1993). The role of attention for the Simon effect. Psychol. Res. 55, 208-222.

Hommel, B. (1996). S-R compatibility effects without response uncertainty. Q. J. Exp. Psychol. 49A, 546-571.

Hommel, B. (2004). Event files: feature binding in and across perception and action. Trends Cogn. Sci. 8, 494-500.

Hommel, B. (2009). Action control according to TEC (theory of event coding). Psychol. Res. 73, 512-526.

Hommel, B., Colzato, L. S., and van den Wildenberg, W. P. M. (2009). How social are task representations. Psychol. Sci. 20, 794-798.

Hommel, B., Müsseler, J., Aschersleben, G., and Prinz, W. (2001). The theory of event coding (TEC): a framework for perception and action planning. Behav. Brain Sci. 24, 849-878.

Kammers, M. P. M., de Vignemont, F., Verhagen, L., and Dijkerman, H. C. (2009). The rubber hand illusion in action. Neuropsychologia 47, 204-211.

Knoblich, G., and Sebanz, N. (2006). The social nature of perception and action. Curr. Dir. Psychol. Sci. 15, 99-104.

Kornblum, S., Hasbroucq, T., and Osman, A. (1990). Dimensional overlap: cognitive basis for stimulus-response
compatibility-A model and taxonomy. Psychol. Rev. 97, 253-270.

Kuhbandner, C., Pekrun, R., and Maier M.A. (2010). The role of positive and negative affect in the "mirroring" of other persons' actions. Cogn. Emot. 24, 1182-1190.

Lam, M.Y., and Chua, R. (2009). Influence of stimulus-response assignment on the joint-action correspondence effect. Psychol. Res. 74, 476-480.

Liepelt, R., and Brass, M. (2010). Top-down modulation of motor priming by belief about animacy. Exp. Psychol. 57, 221-227.

Liepelt, R., Wenke, D., Fischer, R. and Prinz, W. (2011). Trial-totrial sequential dependencies in a social and non-social Simon task. Psychol. Res. doi: 10.1007/ s00426-010-0314-313.

Lloyd, D. M. (2007). Spatial limits on referred touch to an alien limb may reflect boundaries of visuo-tactile peripersonal space surrounding the hand. Brain Cogn. 64, 104-109.

Nicoletti, R., and Umiltà, C. (1989). Splitting visual space with attention. J. Exp. Psychol. Hum. Percept. Perform. 15, 164-169.

Olfield, R. C. (1971). The assessment and analysis of handedness: the Edinburgh Inventory. Neuropsychologia 9, 97-113.

Philipp, A. M., and Prinz, W. (2010). Evidence for a role of the responding agent in the joint compatibility effect. Q. J. Exp. Psychol. 28, 1-13.

Pollick, F. E., Paterson, H. M., Bruderlin, A., and Sanford, A. J. (2001). Perceiving affect from arm movement. Cognition 82, B51-B61.

Prinz, W. (1990). "A common coding approach to perception and action," in Relationships between Perception and Action: Current Approaches, eds O. Neumann and W. Prinz (Berlin: Springer), 167-201.

Ruys, K. I., and Aarts, H. (2010). When competition merges people's behavior: interdependency activates shared action representations. J. Exp. Soc. Psychol. 46, 1130-1133.

Schütz-Bosbach, S., Avenanti, A. Aglioti, S. M., and Haggard, P. (2008). Don't do it! Cortical inhibition and self-attribution during action observation. J. Cogn. Neurosci. 21, 1215-1227.

Sebanz, N., Bekkering, H., and Knoblich, G. (2006). Joint actions: bodies and minds moving together. Trends Cogn. Sci. 10, 70-76.

Sebanz, N., Knoblich, G., and Prinz, W. (2003). Representing others' actions: just like one's own? Cognition 88, B11-B21.

Sebanz, N., Knoblich, G., Stumpf, L., and Prinz, W. (2005). Far from action blind: representation of others' action in individuals with autism. Cogn. Neuropsychol. 22, 433-454. 
Simon, J. R. (1990). "The effects of an irrelevant directional cue on human information processing," in StimulusResponse Compatibility: An Integrated Perspective. Advances in Psychology, Vol. 65, eds R. W. Proctor and T. G. Reeve (Amsterdam: North-Holland), 31-86.

Simon, J. R., and Rudell, A. P. (1967). Auditory S-R compatibility: the effect of an irrelevant cue on information processing. J. Appl. Psychol. 51, 300-304.

Treccani, B., Umiltà, C., and Tagliabue, M. (2006). Simon effect with and without awareness of the accessory stimulus. J.
Exp. Psychol. Hum. Percept. Perform. 32, 268-286.

Tsakiris, M., and Haggard, P. (2005). The rubber hand illusion revisited: visuotactile integration and self-attribution. J. Exp. Psychol. Hum. Percept. Perform. 31, 80-91.

van Steenbergen, H. (2007). Neural Integration of Actions and Their Auditory Effects: An fMRI-Study. Unpublished Master Thesis, Leiden University.

Vlainic, E., Liepelt, R., Colzato, L. S., Prinz, W., and Hommel, B. (2010). The virtual co-actor: the social Simon effect does not rely on online feedback from the other. Front. Psychol. 1:208. doi: 10.3389/fpsyg.2010.00208

Conflict of Interest Statement: The authors declare that the research was conducted in the absence of any commercial or financial relationships that could be construed as a potential conflict of interest.

Received: 15 September 2010; accepted: 21 April 2011; published online: 06 May 2011. Citation: Dolk T, Hommel B, Colzato LS, Schütz-Bosbach S, Prinz W and Liepelt $R$
(2011) How "social" is the social Simon effect? Front. Psychology 2:84. doi: 10.3389/ fpsyg.2011.00084

This article was submitted to Frontiers in Cognition, a specialty of Frontiers in Psychology.

Copyright (c) 2011 Dolk, Hommel, Colzato, Schütz-Bosbach, Prinz and Liepelt. This is an open-access article subject to a nonexclusive license between the authors and Frontiers Media SA, which permits use, distribution and reproduction in other forums, provided the original authors and source are credited and other Frontiers conditions are complied with. 